\title{
Poles of light resonances from unitarized Chiral perturbation Theory and their large $N_{c}$ behavior
}

\author{
J.R.Peláez ${ }^{\dagger}$ \\ Dipartimento di Fisica. Universita' degli Studi and INFN, Firenze, Italy \\ Departamento de Física Teórica II, Universidad Complutense, 28040 Madrid, Spain
}

\begin{abstract}
We have recently completed the one loop calculation of meson-meson scattering within Chiral Perturbation Theory. Once unitarized, these amplitudes provide simultaneously a remarkable description of the resonance region up to $1.2 \mathrm{GeV}$ as well as the low energy region, respecting the chiral symmetry expansion. The position of the poles in these amplitudes is related to the mass and width of the associated resonances that are generated without being explicitly included in the Lagrangian. The spectroscopic nature of these states can then be deduced by studying the behavior of these poles, through the $N_{c}$ dependence of the Chiral Perturbation Theory parameters, which can be obtained from QCD in the large $N_{c}$ limit.
\end{abstract}

I take advantage of the occasion to celebrate the long and fruitful physics carrier of J. Schechter, to whom this workshop is dedicated in his 65th anniversary, to review my work, either recently published or still in progress, which is closely related to some of Joe's relevant contributions.

For many years now, we take QCD as the fundamental theory of strong interactions. Its predictions have been thoroughly tested to great accuracy in the perturbative regime (above 1-2 GeV), where a description in terms of quarks and gluons is possible. However, QCD becomes non-perturbative at low energies and the usual perturbative expansion has to be abandoned in favor of somewhat less systematic approaches in terms of mesons. An exception is the formalism of Chiral Perturbation Theory (ChPT) [1, 2], built as the most general derivative expansion of a Lagrangian containing pions, kaons and the eta. These particles are identified as the Goldstone bosons associated to the spontaneous chiral symmetry breaking of the massless QCD theory. In terms of observables ChPT becomes an expansion in powers of energy or momenta over a scale $4 \pi f_{0} \simeq 1.2 \mathrm{GeV}$. For the processes we are interested in, and due to Lorentz invariance, only even powers of energy and momenta occur in the expansion, which are generically denoted as $O\left(p^{2}\right), O\left(p^{4}\right)$, etc... The quark masses, small compared with typical hadronic scales, are introduced in the same power counting, and give rise to the masses of the $\pi, K$ and $\eta$ mesons, counted as $M=O\left(p^{2}\right)$. The ChPT Lagrangian thus allows for true Quantum Field Theory calculations, including meson loops, whose divergences are renormalized in a set of chiral parameters at each order in the expansion. In particular, the theory is finite at each order and predictive in the sense that once the set of parameters up to a given order is determined from some experiments, these very same parameters can, and should describe, to that order, any other physical process involving mesons. As usual in a renormalization program, the "bare" parameters appearing in the Lagrangian may de- 
pend on an arbitrary regularization scale $\mu$; however, the physical observables are scale independent, since the $\mu$ dependence is canceled through the regularization of the loop integrals. In other words, all the relevant physical scales are those given by parameters in the Lagrangian. Another salient feature of ChPT is its model independence and the fact that it has been possible to relate its parameters to those of QCD, at least in the limit of a large number of colors $N_{c}[2,3]$.

Unfortunately, the applicability of ChPT is limited to low energies or momenta. First, because the number of independent terms allowed by symmetry increases dramatically at each order. Second, because resonances appear rather soon in meson physics and these states are associated to poles in the second Riemann sheet of the amplitudes. Such a nonperturbative behavior cannot be accommodated by truncated series as those of ChPT (as a matter of fact there are also logarithmic terms, but for this discussion, only the powers count). A third way of seeing this problem is the violation of the unitarity constraint, which becomes more and more severe as the energy increases.

During the last few years, unitarization methods have emerged as a powerful tool to extend ChPT to the resonant region $[4,5,6,7]$. The basic point is to realize that the unitarity of meson-meson partial waves, $t$, determines completely the imaginary part of the inverse of the amplitude $\operatorname{Im} 1 / t$. Actually, the dynamical information comes through $\operatorname{Re} 1 / t$, which has to be calculated from theory. The use of ChPT to calculate $\operatorname{Re} 1 / t$ has yielded remarkable results. In particular, we have recently shown [7] that, by unitarizing the one-loop ChPT amplitudes, it is possible to generate the resonant behavior of light meson resonances, while respecting at the same time the low energy chiral expansion (in particular the values of the chiral parameters already determined from other processes).

In what follows we will review all those results and present our recent determination [8] of the pole positions of the generated resonances, which are related to their masses and widths. We will also present an study of the behavior of these poles in the large $N_{c}$ limit. This whole approach is of special interest for the meson spectroscopy community, since these resonances are generated from the most general Lagrangian consistent with QCD, and therefore without any bias toward its existence. Let us recall that in particular the existence of light scalar mesons is still under a strong debate, and the appearance of these states in our work could help shedding some light in this issue. The fact that nine of these scalar poles seem to appear together, suggests that they could form a multiplet. Finally, if the existence of these states is rather controversial, even more so is their nature or composition in terms of quarks and gluons. However, quarks and gluon states are properly defined only in a microscopic framework, and our approach allows for a clear link with QCD in the large $N_{c}$ limit, where these states have a consistent definition.

\section{MESON MESON SCATTERING WITHIN UNITARIZED CHIRAL PERTURBATION THEORY}

Customarily partial waves $t_{I J}$ of definite isospin $I$ and angular momentum $J$ are used to compare with experiment. Omitting for simplicity the $I, J$ subindices, the chiral expansion becomes $t \simeq t_{2}+t_{4}+\ldots$, with $t_{2}$ and $t_{4}$ of $\mathscr{O}\left(p^{2}\right)$ and $\mathscr{O}\left(p^{4}\right)$, respectively. The unitarity relation is rather simple for the partial waves $t_{i j}$, where $i, j$ denote the 
different available states. For instance, when two states, say "1" and "2", are accessible, it becomes

$$
\operatorname{Im} T=T \Sigma T^{*} \Rightarrow \operatorname{Im} T^{-1}=-\Sigma \Rightarrow T=\left(\operatorname{Re} T^{-1}-i \Sigma\right)^{-1}
$$

with

$$
T=\left(\begin{array}{ll}
t_{11} & t_{12} \\
t_{12} & t_{22}
\end{array}\right) \quad, \quad \Sigma=\left(\begin{array}{cc}
\sigma_{1} & 0 \\
0 & \sigma_{2}
\end{array}\right)
$$

where $\sigma_{i}=2 q_{i} / \sqrt{s}$ and $q_{i}$ is the C.M. momentum of the state $i$. Let us remark again that we only need to know the real part of the Inverse Amplitude since the imaginary part is fixed by unitarity. Note that the unitarity relations are non-linear and therefore cannot be satisfied exactly with a perturbative expansion like that of ChPT. Nevertheless, unitarity holds perturbatively, i.e,

$$
\operatorname{Im} T_{2}=0, \quad \operatorname{Im} T_{4}=T_{2} \Sigma T_{2}^{*}+\mathscr{O}\left(p^{6}\right) .
$$

Using in eq.(1) the ChPT expansion of $\operatorname{Re} T^{-1} \simeq T_{2}^{-1}\left(1-\left(\operatorname{Re} T_{4}\right) T_{2}^{-1}+\ldots\right)$ ensures that we will be respecting the ChPT low energy expansion thus and taking into account all the information included in the chiral Lagrangians.

Let us recall that the leading order Lagrangian is universal since it only depends on the chiral symmetry breaking scale $f_{0}$, which corresponds to the pion decay constant in the chiral limit, and the leading order meson masses $M_{\pi}^{0}, M_{K}^{0}$ and $M_{\eta}^{0}$. The dependence on the QCD dynamics only comes through the one loop chiral parameters, which for meson-meson scattering in $\mathrm{SU}(3)$ are called $L_{i}$, with $i=1 \ldots 8$ [2].

As a matter of fact there is an additional simplification that is allowed in ChPT, since the $O\left(p^{6}\right)$ and higher orders in Eq.(3) can be made to vanish if the substitution of $f_{0}$ in terms of the observables $f_{\pi}$ or $f_{K}$ or $f_{\eta}$ is made to match their corresponding powers on both sides of the above equations. In such case:

$$
\operatorname{Im} T_{2}=0, \quad \operatorname{Im} T_{4}=T_{2} \Sigma T_{2}^{*} .
$$

We will call these conditions "exact perturbative unitarity". From eq.(4), we find

$$
T \simeq T_{2}\left(T_{2}-T_{4}\right)^{-1} T_{2},
$$

which is the coupled channel IAM, which we have used to unitarize simultaneously all the one-loop ChPT meson-meson scattering amplitudes [7].

Part of this unitarization program had been carried out first for partial waves in the elastic region, where a single channel is enough to describe the data. In such a way were found the $\rho$ and $\sigma$ poles in $\pi \pi$ scattering and that of $K^{*}$ in $\pi K \rightarrow \pi K$ [4]. The $\kappa$ pole can also be obtained in the elastic single channel formalism but was only noticed later in this context. A simplified coupled channel calculation, considering only the leading order and the dominant s-channel loops, but neglecting crossed and tadpole loop diagrams, was used in [5], since at that time not all the meson-meson amplitudes were known to one-loop. Despite the approximations, it resulted in a remarkable description of meson-meson scattering up to $1.2 \mathrm{GeV}$. The poles associated to the $\rho, K^{*}, f_{0}, a_{0}, \sigma$ and $\kappa$ resonances were found when these partial waves were continued to the second Riemann sheet of the complex energy plane. However, the approximations implied that 
only the leading order of the expansion was recovered at low energies. In addition, the divergences were regularized with a cutoff, which could violate chiral symmetry by introducing an spurious parameter. Furthermore, due to this cutoff regularization, it was not possible to compare the $L_{i}$ parameters, which are supposed to encode the underlying QCD dynamics, with those already present in the literature, obtained from other low energy processes. This, together with a residual cutoff dependence due to the absence of the whole set of one loop diagrams makes it hard to study the large $N_{c}$ behavior that, as we will see is inherited by ChPT from QCD

Since not only the nature, but also the existence of light scalar resonances is rather controversial, it is very relevant to check that these poles and their features are not just artifacts of the approximations, estimate the uncertainties in their parameters, and check their compatibility with other experimental information regarding ChPT.

That is why the $K \bar{K} \rightarrow K \bar{K}$ one-loop amplitudes were calculated in [6]: together with previous workss [9], the [6] results allowed for the unitarization of the $\pi \pi, K \bar{K}$ coupled system. They found good agreement of the IAM description with the existing $L_{i}$, reproducing again the resonances in that system. Finally, we have completed more recently [7] the one-loop meson-meson scattering calculation. There are three new amplitudes: $K \eta \rightarrow K \eta, \eta \eta \rightarrow \eta \eta$ and $K \pi \rightarrow K \eta$, but we have recalculated the other five amplitudes unifying the notation, ensuring “ exact perturbative unitarity”, Eq.(4), and also correcting some errors in the literature. Next, we have applied the coupled channel IAM to these amplitudes. Our results allow for a direct comparison with the standard low-energy chiral parameters, which we find in very good agreement with previous determinations from low-energy data. The main differences with [5] are: i) we consider the full calculation of all the one-loop amplitudes in dimensional regularization, ii) we are able to describe simultaneously the low energy and the resonance regions, and iii) we pay special attention to the estimation of uncertainties.

First of all we checked that the resonant features were still present with the standard ChPT parameters, that we have given in Table 1 . As already commented, this comparison can only be performed now since we have all the amplitudes renormalized in the standard $\overline{M S}-1$ scheme.

In Fig. 1 we show the results. Let us recall that meson-meson scattering data are very hard to obtain, and usually have large systematic uncertainties, which are the largest contribution to the error bands of the curves in the figure as well as in the parameters in Table 1. As it happened in [7], the uncertainty bands are calculated from a MonteCarlo Gaussian sampling (1000 points) of the $L_{i}$ sets within their error bars, assuming they are uncorrelated.

As a matter of fact in Table 1 we give three sets of parameters for the IAM, reasonably compatible among them and with standard ChPT parameters. The IAM I fit was obtained reexpressing all the $f_{0}$ appearing in the amplitudes by their expression in terms of just $f_{\pi}$, which. although simpler, is somewhat unnatural when dealing with kaons or etas. The plots and the uncertainties of this fit were already given in [7] and therefore the corresponding plots are not shown here. There, it could be observed that the description of the $f_{0}(980)$ region was somewhat poor, yielding a too small width for the resonance.

That is the reason why in Fig.1 we present the results [8] using amplitudes written in terms of $f_{K}$ and $f_{\eta}$ when dealing with processes involving kaons or etas, that we call set IAM II. As it can be noticed, the data in the $f_{0}(980)$ region is well within the 
uncertainties. In particular, we have rewritten our $O\left(p^{2}\right)$ amplitudes changing one factor of $1 / f_{\pi}$ by $1 / f_{K}$ for each two kaons present between the initial or final state, or by $1 / f_{\eta}$ for each two etas appearing between the initial and final states. In the special case $K \eta \rightarrow K \pi$ we have changed $1 / f_{\pi}^{2}$ by $1 /\left(f_{K} f_{\eta}\right)$. Of course, these changes introduce some corrections at $O\left(p^{4}\right)$ which can be easily obtained using the relations between the decay constants and $f_{0}$ provided in $[2,7]$. The $1 / f_{\pi}$ factor in each loop function at $O\left(p^{4}\right)$ (generically, the $J(s)$ given in the appendix of [7]) have to be changed according to eqs.(4), that is, they satisfy the "exact perturbative unitarity" condition and are the same as the previous ones, up to $O\left(p^{6}\right)$ differences. However, at high energies there can be some numerical differences. Note that the only parameters that suffer a sizable change are those related to the definition of decay constants: $L_{4}$ and $L_{5}$. We also give in Table 1 a third fit, IAM III, obtained as IAM II but fixing $L_{4}$ to zero as in the most recent $K_{14}$ $O\left(p^{4}\right)$ determinations. This is the value estimated from the large $N_{c}$ limit, and since our fits are not very sensitive to the variations in $L_{4}$ in this way we avoid that it could get an unnatural value that correspond just to a very tiny improvement of the $\chi^{2}$.

TABLE 1. Different sets of chiral parameters $\left(\times 10^{3}\right)$. The first column comes from recent analysis of $K_{l 4}$ decays [10] $\left(L_{4}\right.$ and $L_{6}$ are set to zero). In the ChPT column $L_{1}, L_{2}, L_{3}$ come from [11] and the rest from [2]. The three last ones correspond to the values from the IAM including the uncertainty due to different systematic error used on different fits. Sets II and III are obtained using amplitudes expressed in terms of $f_{\pi}, f_{K}$ and $f_{\eta}$, whereas the amplitudes in set I are expressed in terms of $f_{\pi}$ only.

\begin{tabular}{c||c|c||c|cc|}
\hline Parameter & $K_{l 4}$ decays & ChPT & IAM I & IAM II & IAM III \\
\hline$L_{1}^{r}\left(M_{\rho}\right)$ & 0.46 & $0.4 \pm 0.3$ & $0.56 \pm 0.10$ & $0.59 \pm 0.08$ & $0.60 \pm 0.09$ \\
$L_{2}^{r}\left(M_{\rho}\right)$ & 1.49 & $1.35 \pm 0.3$ & $1.21 \pm 0.10$ & $1.18 \pm 0.10$ & $1.22 \pm 0.08$ \\
$L_{3}$ & -3.18 & $-3.5 \pm 1.1$ & $-2.79 \pm 0.14$ & $-2.93 \pm 0.10$ & $-3.02 \pm 0.06$ \\
$L_{4}^{r}\left(M_{\rho}\right)$ & 0 (fixed) & $-0.3 \pm 0.5$ & $-0.36 \pm 0.17$ & $0.2 \pm 0.004$ & 0 (fixed) \\
$L_{5}^{r}\left(M_{\rho}\right)$ & 1.46 & $1.4 \pm 0.5$ & $1.4 \pm 0.5$ & $1.8 \pm 0.08$ & $1.9 \pm 0.03$ \\
$L_{6}^{r}\left(M_{\rho}\right)$ & 0 (fixed) & $-0.2 \pm 0.3$ & $0.07 \pm 0.08$ & $0 \pm 0.5$ & $-0.07 \pm 0.20$ \\
$L_{7}$ & -0.49 & $-0.4 \pm 0.2$ & $-0.44 \pm 0.15$ & $-0.12 \pm 0.16$ & $-0.25 \pm 0.18$ \\
$L_{8}^{r}\left(M_{\rho}\right)$ & 1.00 & $0.9 \pm 0.3$ & $0.78 \pm 0.18$ & $0.78 \pm 0.7$ & $0.84 \pm 0.23$ \\
\hline
\end{tabular}

TABLE 2. Scattering lengths $a_{I J}$ and slope parameters $b_{I J}$ for different meson-meson scattering channels. For experimental references see [7]. Let us remark that our one-loop IAM results at threshold are very similar to those of two-loop ChPT.

\begin{tabular}{|c|c|c|c|c|}
\hline $\begin{array}{c}\text { Threshold } \\
\text { parameter }\end{array}$ & Experiment & $\begin{array}{c}\text { IAM fit I } \\
{[7]}\end{array}$ & $\begin{array}{c}\text { ChPT } \mathscr{O}\left(p^{4}\right) \\
{[4,9]}\end{array}$ & $\begin{array}{c}\text { ChPT } \mathscr{O}\left(p^{6}\right) \\
{[12]}\end{array}$ \\
\hline \hline$a_{00}$ & $0.26 \pm 0.05$ & $0.231_{-0.006}^{+0.003}$ & 0.20 & $0.219 \pm 0.005$ \\
$b_{00}$ & $0.25 \pm 0.03$ & $0.30 \pm 0.01$ & 0.26 & $0.279 \pm 0.011$ \\
$a_{20}$ & $-0.028 \pm 0.012$ & $-0.0411_{-0.001}^{+0.0009}$ & -0.042 & $-0.042 \pm 0.01$ \\
$b_{20}$ & $-0.082 \pm 0.008$ & $-0.074 \pm 0.001$ & -0.070 & $-0.0756 \pm 0.0021$ \\
$a_{11}$ & $0.038 \pm 0.002$ & $0.0377 \pm 0.0007$ & 0.037 & $0.0378 \pm 0.0021$ \\
$a_{1 / 20}$ & $0.13 \ldots 0.24$ & $0.11_{-0.09}^{+0.06}$ & 0.17 & \\
$a_{3 / 20}$ & $-0.13 \ldots-0.05$ & $-0.049_{-0.003}^{+0.002}$ & -0.5 & \\
$a_{1 / 21}$ & $0.017 \ldots 0.018$ & $0.016 \pm 0.002$ & 0.014 & \\
$a_{10}$ & & $0.15_{-0.11}^{+0.07}$ & 0.0072 & \\
\hline
\end{tabular}



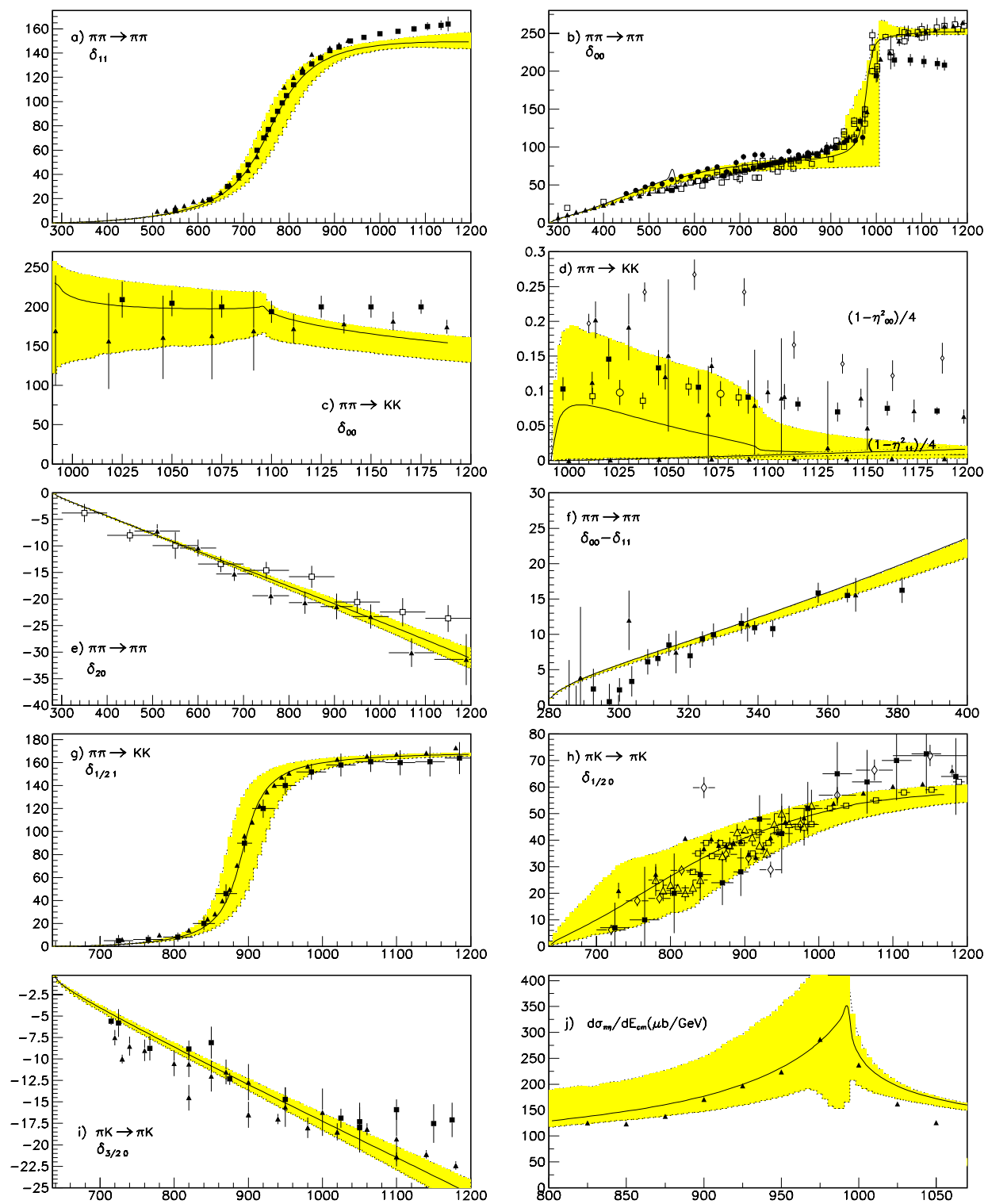

FIGURE 1. IAM fit to meson-meson scattering data, set II in Table 1. The uncertainties cover the estimated systematic errors. The statistical errors from the fit would be much smaller. The data comes from [13] 
In conclusion, we have a set of amplitudes obtained from unitarized ChPT that describe meson-meson scattering data up to energies of $1.2 \mathrm{GeV}$. That is, they describe the resonant region while respecting simultaneously the low energy constraints, ensuring a remarkable low energy description, as can be seen from the values of the the scattering lengths and slopes listed in Table 2. In addition, we have checked that the chiral parameters needed for this unitarized description are compatible with those from standard ChPT, and, since the expressions are fully renormalized, we are not including any dependence on any spurious parameter.

\section{T-MATRIX POLES ASSOCIATED TO RESONANCES}

Thus, with the amplitudes just commented we will now study whether we still obtain the same poles as obtained with previous approaches. The $\sigma$ and $\kappa$ states are still rather controversial, although their poles have been found by several groups (that of Joe Schechter among others) with different approaches [14, 15]. In addition, recent experiments seem to require such poles [16], supporting strongly their existence since they have completely different systematics compared with meson scattering processes.

The poles in unitarized chiral amplitudes have triggered the interest in the hadron spectroscopy community since they are not included in the ChPT Lagrangian and are therefore generated without any theoretical bias toward their existence, classification in multiplets, or nature. Since the scalar resonances $\sigma, \kappa, a_{0}(980)$ and $f_{0}(980)$ appear together in these chiral symmetric approaches, it seems very natural to interpret that they belong to the same nonet. Nevertheless, we should distinguish two types of resonances: already in [17] it was noted that the scalars could be generated with just a cutoff, that is, without the need to include one-loop chiral parameters, in contrast with the vector mesons, which needed these parameters, particularly $L_{1}, L_{2}$ and $L_{3}$ [5]. The vectors seem to be fairly well established as $q \bar{q}$ states, and this difference hints that the lightest scalar nonet may have a different nature. With the amplitudes described in the previous section we expect to reach a more conclusive statement, since they respect the chiral expansion, and, being completely renormalized, have no spurious dependence on any cutoff or dimensional regularization scale.

Thus, we show in Table 3 the poles and their uncertainties, for the different IAM sets of parameters given in Table 1, compared with those obtained in the "approximated" IAM [5]. For illustration we show in Table 4 the poles listed presently in the PDG [18]. Let us comment briefly these results for the different resonances:

- The vectors $\rho(770)$ and $K^{*}(892)$, are very stable in the chiral unitary approaches. They appear consistently in single channel approaches [4], approximated coupled channel IAM [5] and the complete IAM [7].

- The $\sigma$ and $\kappa$ poles are robust within these approaches. They appear with compatible values in single channel approaches, approximated coupled channel IAM and the complete IAM. Note the small uncertainties in some of their parameters.

- The $f_{0}(980)$ mass is robust, and the pole appears both in the approximated and complete IAM approaches (of course it cannot appear in the single channel case since it has a sizable decay to two different channels). However, we have already 
remarked that the unitarized ChPT amplitudes using just $f_{\pi}$ as those given in [7], yield a somewhat poor description of its width. Nevertheless is well described when using $f_{\pi}, f_{K}$ and $f_{\eta}$.

- Although the data in the $a_{0}(980)$ region are always well described, the presence of a pole is strongly dependent on how the unitarized ChPT amplitudes are parametrized. There is no pole when using only $f_{\pi}$, which was already pointed out in [19], where, using the "approximated" coupled channel IAM with just $f_{\pi}$, a "cusp" interpretation of the $a_{0}(980)$ enhancement in $\pi \eta$ production was suggested. However, we do find a pole when using $f_{\pi}, f_{K}$ and $f_{\eta}$ either with the approximated or complete IAM. Thus, this pole is rather unstable as can be noticed from its large uncertainties in Table 3. We are somewhat more favorable toward the pole interpretation because the use of $f_{\pi}, f_{K}$ and $f_{\eta}$ is more natural and is also able to describe better the $f_{0}(980)$ width.

Let us nevertheless note that the $f_{0}(980)$ and $a_{0}(980)$ resonances are very close to the $K \bar{K}$ threshold, and they couple strongly to this state. The proximity of this threshold can produce a considerable distortion in the resonance shape, whose relation to the pole position can then be far from the expected one for narrow resonances. In addition these states have a large mass ad it is likely that their nature should be understood from a mixture with heavier states.

TABLE 3. Pole positions (with errors) in meson-meson scattering. When close to the real axis the mass and width of the associated resonance is $\sqrt{s_{\text {pole }}} \simeq M-i \Gamma / 2$.

\begin{tabular}{|c|c|c|c|c|c|c|}
\hline$\sqrt{s_{\text {pole }}}(\mathrm{MeV})$ & $\rho$ & $K^{*}$ & $\sigma$ & $f_{0}$ & $a_{0}$ & $\kappa$ \\
\hline $\begin{array}{l}\text { IAM Approx } \\
\text { (no errors) }\end{array}$ & 759-i 71 & $892-\mathrm{i} 21$ & $442-\mathrm{i} 227$ & 994-i 14 & $1055-\mathrm{i} 21$ & $770-\mathrm{i} 250$ \\
\hline $\begin{array}{c}\text { IAM I } \\
\text { (errors) }\end{array}$ & $\begin{array}{c}760-\mathrm{i} 82 \\
\pm 52 \pm \mathrm{i} 25\end{array}$ & $\begin{array}{c}886-\mathrm{i} 21 \\
\pm 50 \pm \mathrm{i} 8\end{array}$ & $\begin{array}{c}443-\mathrm{i} 217 \\
\pm 17 \pm \mathrm{i} 12\end{array}$ & $\begin{array}{c}988-\mathrm{i} 4 \\
\pm 19 \pm \mathrm{i} 3\end{array}$ & cusp? & $\begin{array}{l}750-\mathrm{i} 226 \\
\pm 18 \pm \mathrm{i} 11\end{array}$ \\
\hline $\begin{array}{l}\text { IAM II } \\
\text { (errors) }\end{array}$ & $\begin{array}{c}754-\mathrm{i} 74 \\
\pm 18 \pm \mathrm{i} 10 \\
\end{array}$ & $\begin{array}{c}889-\mathrm{i} 24 \\
\pm 13 \pm \mathrm{i} 4 \\
\end{array}$ & $\begin{array}{l}440-\mathrm{i} 212 \\
\pm 8 \pm \mathrm{i} 15 \\
\end{array}$ & $\begin{array}{l}973-\mathrm{i} 11 \\
+39+i 189 \\
-127-i 11 \\
\end{array}$ & $\begin{array}{l}1117-\mathrm{i} 12 \\
+24+i 43 \\
-320-i 12 \\
\end{array}$ & $\begin{array}{l}753-\mathrm{i} 235 \\
\pm 52 \pm \mathrm{i} 33 \\
\end{array}$ \\
\hline $\begin{array}{c}\text { IAM III } \\
\text { (errors) }\end{array}$ & $\begin{array}{c}748-\mathrm{i} 68 \\
\pm 31 \pm \mathrm{i} 29\end{array}$ & $\begin{array}{c}889-\mathrm{i} 23 \\
\pm 22 \pm \mathrm{i} 8 \\
\end{array}$ & $\begin{array}{l}440-\mathrm{i} 216 \\
\pm 7 \pm \mathrm{i} 18\end{array}$ & $\begin{array}{c}972-\mathrm{i} 8 \\
+21 \pm \mathrm{i} 7 \\
-56\end{array}$ & $\begin{array}{r}1091-i 52 \\
+19+i 21 \\
-45-i 40 \\
\end{array}$ & $\begin{array}{l}754-\mathrm{i} 230 \\
\pm 22 \pm \mathrm{i} 27 \\
\end{array}$ \\
\hline
\end{tabular}

TABLE 4. Mass and widths or pole positions of the light resonances quoted in the PDG [18]. Recall that for narrow resonances $\sqrt{s_{\text {pole }}} \simeq M-i \Gamma / 2$

\begin{tabular}{ccccccc}
\hline PDG2002 & $\rho(770)$ & $K^{*}(892)^{ \pm}$ & $\sigma$ or $f_{0}(600)$ & $f_{0}(980)$ & $a_{0}(980)$ & $\kappa$ \\
\hline Mass $(\mathrm{MeV})$ & $771 \pm 0.7$ & $891.66 \pm 0.26$ & $(400-1200)-\mathrm{i}(300-500)$ & $980 \pm 10$ & $980 \pm 10$ & not \\
Width $(\mathrm{MeV})$ & $149 \pm 0.9$ & $50.8 \pm 0.9$ & (we list the pole) & $40-100$ & $50-100$ & listed \\
\hline
\end{tabular}

\section{CHIRAL PERTURBATION THEORY AND THE LARGE $N_{C}$}

QCD is not perturbative at low energies, say below 1 or $2 \mathrm{GeV}$. In this region, however, the limit of a large number of colors $N_{c}$, despite $N_{c}$ being only 3 in nature, has emerged as a powerful tool to understand many qualitative features of QCD and also as a guiding 
line to organize calculations [20]. This is one more topic that Joe has addressed in his long career [20]. The advantage of the large $N_{c}$ limit to study resonances is that $q \bar{q}$ states become real bound states as $N_{c} \rightarrow \infty$. In particular, it has been shown that their mass should be basically constant $M \simeq O(1)$, whereas their decay width to two mesons should decrease as $\Gamma \simeq O\left(1 / N_{c}\right)$. A similar behavior should hold for glueballs decaying to two mesons. In contrast, some multiquark states as $q q \bar{q} \bar{q}$ are expected to become simply unbound, that is, the meson-meson continuum [21].

ChPT inherits the large $N_{c}$ features of QCD through the meson masses and decay constants, that behave as $f_{\pi}, f_{K}, f_{\eta}=O\left(\sqrt{N_{c}}\right)$ and $M_{\pi}, M_{K}, M_{\eta}=O(1)$, and also through the scaling of the chiral parameters [3], that we list in Table 5. There are also estimates of the value for $N_{c}=3$, also listed in Table 5. One must bear in mind that there is an uncertainty on the renormalization scale where these estimates should be evaluated, although on general grounds one expects them to be valid for $\mu \simeq 0.5-1 \mathrm{GeV}$. From Table 5 we see that, despite 3 is not such a large number and the scale uncertainty, the agreement with both ChPT and the IAM parameters is fairly impressive.

TABLE 5. Different sets of chiral parameters $\left(\times 10^{3}\right)$. For illustration, the ChPT and IAM I columns are repeated from Table 1. Other IAM sets give similar results. In the third column we give the leading large $N_{c}$ estimates for the chiral parameters, with $N_{c}$ set to 3 . The last column shows the leading large $N_{c}$ behavior, calculated from QCD.

\begin{tabular}{|c|c|c|c|c|}
\hline $\begin{array}{r}\text { Parameter } \\
\times 10^{-3}\end{array}$ & $\begin{array}{c}\text { ChPT } \\
\mu=770 \mathrm{MeV}\end{array}$ & $\begin{array}{c}\text { IAM I } \\
\mu=770 \mathrm{MeV}\end{array}$ & $\begin{array}{c}\text { Large } N_{c}, \\
N_{c}=3\end{array}$ & $\begin{array}{l}\text { Large } N_{c} \\
\text { behavior }\end{array}$ \\
\hline $2 L_{1}-L_{2}$ & $-0.6 \pm 0.2$ & $0.56 \pm 0.10$ & 0 & $O(1)$ \\
\hline$L_{2}$ & $1.35 \pm 0.3$ & $1.21 \pm 0.10$ & 1.8 & $O\left(N_{c}\right)$ \\
\hline$L_{3}^{2}$ & $-3.5 \pm 1.1$ & $-2.79 \pm 0.14$ & -4.3 & $O\left(N_{c}\right)$ \\
\hline$L_{4}$ & $-0.3 \pm 0.5$ & $-0.36 \pm 0.17$ & 0 & $O(1)$ \\
\hline$L_{5}^{4}$ & $1.4 \pm 0.5$ & $1.4 \pm 0.5$ & 2.1 & $O\left(N_{c}\right)$ \\
\hline$L_{6}$ & $-0.2 \pm 0.3$ & $0.07 \pm 0.08$ & 0 & $O(1)$ \\
\hline$L_{7}^{0}$ & $-0.4 \pm 0.2$ & $-0.44 \pm 0.15$ & -0.3 & $O(1)$ \\
\hline$L_{8}^{\prime}$ & $0.9 \pm 0.3$ & $0.78 \pm 0.18$ & 0.8 & $O\left(N_{c}\right)$ \\
\hline
\end{tabular}

\section{THE LARGE $N_{C}$ BEHAVIOR OF THE LIGHTEST RESONANCES}

The fact that ChPT is the most general Lagrangian compatible with the QCD chiral symmetry breaking, and in particular that it inherits the large $N_{c}$ features of QCD, will allow us to study the large $N_{c}$ behavior of these resonances and get a hint on their nature. We will simply impose the scaling of the ChPT parameters at $\mu=770 \mathrm{MeV}$ in the IAM amplitudes fitted to the data, which therefore correspond to $N_{c}=3$. For narrow resonances the pole position is related to the mass and width of a given resonance by $\sqrt{s_{\text {pole }}} \simeq M-i \Gamma / 2$. By comparing the actual behavior of the poles with that expected from large $N_{c}$ we could, in principle, elucidate the nature (or at least the dominant component) of the resonant states. Already in [15] it was shown, using unitarized mesonmeson scattering from a chiral resonance lagrangian and only the s-loops unitarized with an N/D method, that the lightest scalar resonances did not behave as one would expect 

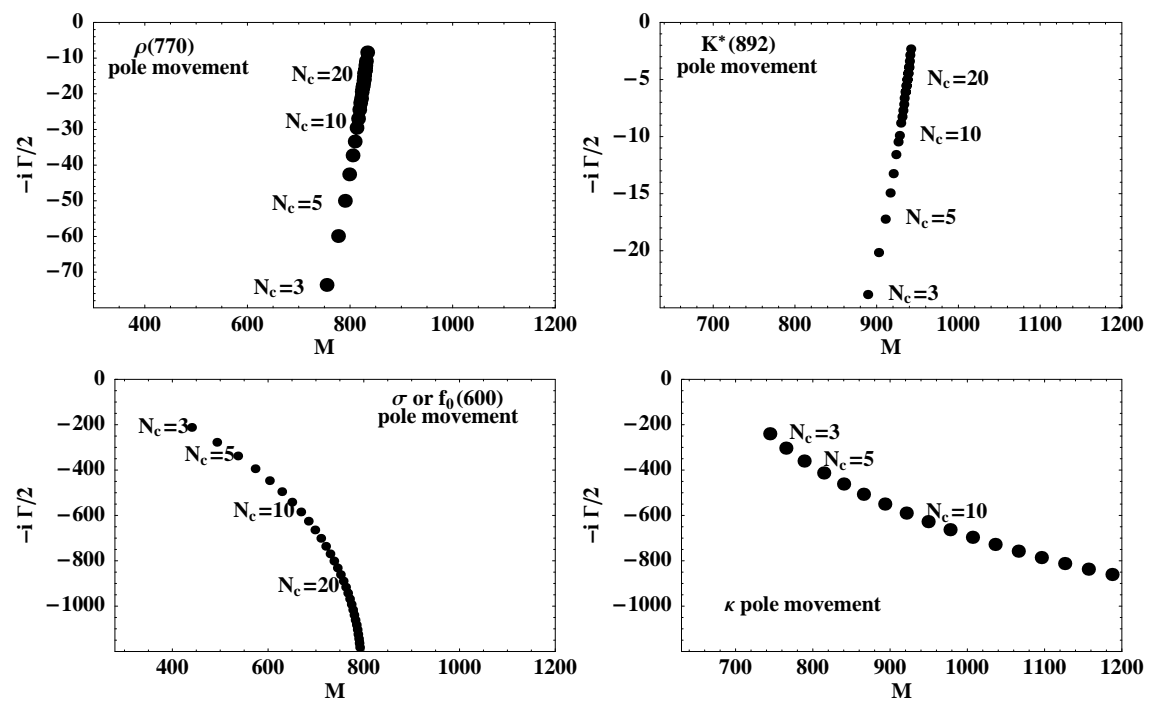

FIGURE 2. Large $N_{c}$ dependence of the pole positions in the lower half of the second Riemann $\sqrt{s}$ sheet of the meson meson scattering amplitude, obtained from the unitarized one-loop Chiral Perturbation Theory calculation. For each value of $N_{c}$ the pole is represented by a dot, in different meson-meson scattering channel. Note that the $\sigma$ and $\kappa$ behavior is opposite to that of well know vector states as the $\rho$ and $K^{*}$.

for $\bar{q} q$ states. We will next show the results of our, still preliminary, study with the unitarized ChPT renormalized amplitudes.

In Fig. 2 we thus show the displacement of the poles in several channels of meson meson scattering. Each dot corresponds to a different value of $N_{c}$. First of all, we want to test the method, and for that we turn to vector resonances, which are well established $q \bar{q}$ states. Thus, on the top left, we represent the $I, J=1,1$ channel of $\pi \pi$ scattering and thus the movement of the $\rho(770)$ pole as $N_{c}$ increases. On the top right we display the same but for $\pi K$ for $I, J=1,1 / 2$, that is, the $K^{*}(982)$ movement. Remarkably, in both cases we can notice that their mass tends to a constant and that the width decreases as $1 / N_{c}$, as expected for $q \bar{q}$ states.

Once we have checked that this approach reproduces correctly the expected behavior of $q \bar{q}$ states, we turn to the controversial scalar resonances. On the bottom left of Fig.2 we represent the movement of the pole commonly associated to the $\sigma$ and on the right, that associated with the $\kappa$ resonance in $\pi K$ scattering. For simplicity we keep the notation $\sqrt{s_{\text {pole }}} \simeq M-i \Gamma / 2$, although these poles are so far from the real axis that the interpretation in terms of mass and width is no longer straightforward. Note that the behavior of both the $\sigma$ and $\kappa$ poles is completely at variance with that expected for $q \bar{q}$ states. In particular, both $M$ and $\Gamma$ grow with $N_{c}$ for both states. This behavior rules out both the $q \bar{q}$ and glueball interpretations. Let us remark, however, that the four-quark state interpretation (also two-meson molecules) [21] is able to accomodate the fact that these states become the meson-meson continuum as $N_{c}$ grows. 


\section{CONCLUSION AND OUTLOOK}

We have shown that unitarized meson-meson scattering ChPT amplitudes provide a simultaneous description of the low energy and resonant regions below $1.2 \mathrm{GeV}$. This description respects the chiral symmetry constraints, and in particular the low energy chiral expansion up to next to leading order. It is also renormalization scale independent, thus avoiding any spurious dependence from artificial scales. We have seen that the unitarized fit leads to parameters compatible with those of standard ChPT and that it yields scattering lengths compatible with higher order calculations and the most recent low energy data.

With these amplitudes we have shown that all the resonant shapes are reproduced, and that their description in terms of poles is robust, with the possible exception of the $a_{0}$ (that could alternatively be interpreted as a cusp). We have provided uncertainties for the determinations of these poles. Since our amplitudes are built from chiral symmetry and unitarity in a very general way, those states which are robust under different unitarization techniques, seem to be an unavoidable requirement of chiral symmetry and unitarity.

We have also presented an on-going study of the large $N_{c}$ behavior of the poles generated with the IAM and ChPT. The behavior of the vectors follows remarkably well the expected behavior, given its established $q \bar{q}$ nature. In contrast, the $\sigma$ and $\kappa$ states, behave in a completely different way, which disfavors a $q \bar{q}$ or glueball interpretation. A $q q \bar{q} \bar{q}$ interpretation is qualitatively adequate to explain the observed behavior.

However, as already remarked, this study is still preliminary. At present, we are finishing the study of the large $N_{c}$ behavior of the $f_{0}$ and $a_{0}$ poles, for which the situation is more cumbersome due to the larger uncertainty in the $a_{0}$ interpretation from unitarized amplitudes, the proximity of thresholds and possible mixings with more massive multiplets ${ }^{1}$. We are also estimating the errors due to the uncertainty in the renormalization scale where the large $N_{c}$ scaling is imposed. Finally, the interpretation of wide resonances is somewhat fishy in terms of poles and for that reason we are presently looking directly at the large $N_{c}$ behavior of the amplitude (the phase shift) in the physical region. The results will be presented elswhere soon [23], hoping they could be of use in clarifying the spectroscopic status of light meson scalars in the near future.

\section{ACKNOWLEDGMENTS}

First of all, I wish to thank the MRST'03 organizers, and specially A. Fariborz, for their kind invitation and for their efforts to offer us such a pleasant and lively workshop in Syracuse. In addition, I wish to thank A. Gómez Nicola for his comments and his careful reading of the manuscript. I also thank A. Andrianov, E. Espriu, F. Kleefeld for encouraging me to look for the large $N_{c}$ behavior of the poles, as well as R. Jaffe for his comments on the large $N_{c}$ behavior of multiquark hadrons. Work supported by a Marie Curie fellowship, contract MCFI-2001-01155, the Spanish CICYT projects,

\footnotetext{
${ }^{1}$ While preparing this work, an study of the mass matrix of scalar resonances that survive in the large $N_{c}$ has appeared [22], suggesting that the $f_{0}$ and the $a_{0}$ could be such kind of states.
} 
FPA2000-0956, PB98-0782 and BFM2000-1326, the CICYT-INFN collaboration grant 003P 640.15, and the E.U. EURIDICE network contract no. HPRN-CT-2002-00311.

\section{REFERENCES}

1. S. Weinberg, Physica A96 (1979) 327. J. Gasser and H. Leutwyler, Annals Phys. 158 (1984) 142.

2. J. Gasser and H. Leutwyler, Nucl. Phys. B 250 (1985) 465.

3. A. A. Andrianov, Phys. Lett. B 157, 425 (1985). A. A. Andrianov and L. Bonora, Nucl. Phys. B 233, 232 (1984). D. Espriu, E. de Rafael and J. Taron, Nucl. Phys. B 345 (1990) 22 [Erratum-ibid. B 355 (1991) 278]. S. Peris and E. de Rafael, Phys. Lett. B 348 (1995) 539 [arXiv:hep-ph/9412343].

4. T. N. Truong, Phys. Rev. Lett. 61 (1988) 2526. Phys. Rev. Lett. 67, (1991) 2260; A. Dobado, M.J.Herrero and T.N. Truong, Phys. Lett. B235 (1990) 134. A. Dobado and J. R. Pelaez, Phys. Rev. D 47 (1993) 4883. Phys. Rev. D 56 (1997) 3057.

5. J. A. Oller, E. Oset and J. R. Pelaez, Phys. Rev. Lett. 80 (1998) 3452; Phys. Rev. D 59 (1999) 074001 [Erratum-ibid. D 60 (1999) 099906]. J. A. Oller, E. Oset and J. R. Peláez, Phys. Rev. D 62 (2000) 114017.

6. F. Guerrero and J. A. Oller, Nucl. Phys. B 537 (1999) 459 [Erratum-ibid. B 602 (2001) 641].

7. A. Gómez Nicola and J. R. Peláez, Phys. Rev. D 65 (2002) 054009

8. J. R. Pelaez and A. Gomez Nicola, AIP Conf. Proc. 660 (2003) 102 [arXiv:hep-ph/0301049].

9. V. Bernard, N. Kaiser, U.G. Meissner, Phys. Rev. D43 (1991) 2757; Nucl. Phys. B357 (1991) 129; Phys. Rev. D44 (1991) 3698.

10. G. Amorós, J. Bijnens and P. Talavera, Nucl. Phys. B602 (2001) 87.

11. J. Bijnens, G. Colangelo and J. Gasser, Nucl. Phys. B427 (1994) 427.

12. G. Amoros, J. Bijnens and P. Talavera, Nucl. Phys. B 585 (2000) 293 [Erratum-ibid. B 598 (2001) 665].

13. S. D. Protopopescu et al., Phys. Rev. D7, (1973) 1279; P. Estabrooks and A.D.Martin, Nucl.Phys.B79, (1974) 301. G. Grayer et al., Nucl. Phys. B75, (1974) 189. D. Cohen, Phys. Rev. D22, (1980) 2595. W. Hoogland et al., Nucl. Phys. B126 (1977) 109. M. J. Losty et al., Nucl. Phys. B69 (1974) 185. R. Mercer et al., Nucl. Phys. B32 (1971) 381. P. Estabrooks et al., Nucl. Phys. B133 (1978) 490. H. H. Bingham et al., Nucl. Phys. B41 (1972) 1. S. L. Baker et al., Nucl. Phys. B99 (1975) 211. D. Aston et al. Nucl. Phys. B296 (1988) 493. D. Linglin et al., Nucl. Phys. B57 (1973) 64 .S. Pislak et al. [BNL-E865 Collaboration], Phys. Rev. Lett. 87 (2001) 221801.

14. R.L. Jaffe, Phys. Rev. D15 267 (1977); Phys. Rev. D15, 281 (1977). E. van Beveren et al. Z. Phys. C30, 615 (1986). R. Kaminski, L. Lesniak and J. P. Maillet, Phys. Rev. D 50 (1994) 3145. M. Harada, F. Sannino and J. Schechter, Phys. Rev. D 54 (1996) 1991 R. Delbourgo and M. D. Scadron, Mod. Phys. Lett. A 10 (1995) 251. S. Ishida et al., Prog. Theor. Phys. 95 (1996) 745. N. A. Tornqvist and M. Roos, Phys. Rev. Lett. 76 (1996) 1575. S. Ishida et al, Prog. Theor. Phys. 98,621 (1997). D. Black, A. H. Fariborz, F. Sannino, J. Schechter. Phys. Rev. D58:054012,1998. E. van Beveren and G. Rupp, Eur. Phys. J. C 22 (2001) 493 E. van Beveren and G. Rupp, arXiv:hep-ph/0201006.

15. J. A. Oller and E. Oset, Phys. Rev. D 60 (1999) 074023 [arXiv:hep-ph/9809337].

16. E791 Collaboration, Phys. Rev. Lett. 86,(2001) 770. E. M. Aitala et al. [E791 Collaboration], Phys. Rev. Lett. 89 (2002) 121801 [arXiv:hep-ex/0204018]. C. Gobel for the E791 Collab. hep-ex/0012009.

17. J. A. Oller and E. Oset, Nucl. Phys. A 620 (1997) 438 [Erratum-ibid. A 652 (1999) 407] [arXiv:hep$\mathrm{ph} / 9702314]$.

18. K. Hagiwara et al., Phys. Rev. D 66, 010001 (2002).

19. M. Uehara, arXiv:hep-ph/0204020.

20. G. 't Hooft, Nucl. Phys. B 72 (1974) 461. C. Rosenzweig, J. Schechter and C. G. Trahern, Phys. Rev. D 21 (1980) 3388. E. Witten, Annals Phys. 128 (1980) 363.

21. R. L. Jaffe, Proceedings of the Intl. Symposium on Lepton and Photon Interactions at High Energies. Physikalisches Institut, University of Bonn (1981) . ISBN: 3-9800625-0-3

22. V. Cirigliano, G. Ecker, H. Neufeld and A. Pich, arXiv:hep-ph/0305311.

23. J. R. Peláez, in preparation. 
Copyright of AIP Conference Proceedings is the property of American Institute of Physics and its content may not be copied or emailed to multiple sites or posted to a listserv without the copyright holder's express written permission. However, users may print, download, or email articles for individual use. 